Access to this work was provided by the University of Maryland, Baltimore County (UMBC)

ScholarWorks@UMBC digital repository on the Maryland Shared Open Access (MD-SOAR) platform.

Please provide feedback

Please support the ScholarWorks@UMBC repository by emailing scholarworks-group@umbc.edu and telling us what having access to this work means to you and why it's important to you. Thank you. 


\title{
Optomechanically-induced frequency combs
}

\author{
Mohammad-Ali Miri, Giuseppe D'Aguanno, and Andrea Alu* \\ Department of Electrical and Computer Engineering, The University of Texas at Austin, Austin, Texas 78712, USA \\ *alu@mail.utexas.edu
}

\begin{abstract}
We investigate the classical nonlinear dynamics of a single mode optomechanical cavity, showing that a frequency comb made of equidistant spectral lines can be formed in the instability regime due to the excitation of multiple sidebands.

(C)2017 Optical Society of America
\end{abstract}

OCIS codes: 190.0190 (Nonlinear optics), 200.4880 (Optomechanics), 140.3948 (Microcavity devices).

High quality factor optical micro-/nano-cavities with small mode volumes have enabled a regime where optical forces can dominate thermal effects, resulting in strong coupling between the optical and mechanical degrees of freedom. These systems are generally referred to as optomechanical cavities [1]. The possibility of coupling optical and mechanical oscillations on a photonic chip has led to a range of applications in quantum and classical optics including, for example, parametric wavelength conversion [2], reconfigurable optical filtering [3], as well as breaking the reciprocity of light [4]-[6]. An interesting aspect of cavity optomechanical systems is the presence of a giant Kerr-type nonlinearity induced by the radiation pressure force, which is proportional to the optical intensity.

Here, we show that optomechaically-induced nonlinear effects can generate a frequency comb. The comb is formed in the instability regime of the optomechanical system, where the mechanical parametric oscillations generate multiple sidebands. The comb is made of equi-distant spectral lines whose spectral separation coincides with the mechanical resonance frequency. In order to explore this phenomenon, we consider an optomechanical microcavity, as shown in Fig. 1(a), where the interaction between a single pair of coupled optical and mechanical modes is analyzed. The dynamics of such system can be described through the coupled mode equations [1]

$$
\begin{gathered}
\frac{d a}{d t}=\left(i(\Delta+G x)-\frac{\kappa}{2}\right) a+\sqrt{\kappa_{e}} S_{\mathrm{in}}, \\
\frac{d^{2} x}{d t^{2}}=-\Omega_{m}^{2} x-\Gamma_{m} \frac{d x}{d t}+\frac{\hbar G}{m}|a|^{2} .
\end{gathered}
$$

In the above equations, $|a|^{2}$ represents the number of photons stored in the optical mode, $\left|s_{\text {in }}\right|^{2}$ is the input photon flux, $\kappa=\kappa_{e}+\kappa_{\ell}$ represents the sum of external and internal optical losses, $\Delta=\omega_{L}-\omega_{0}$ is the detuning of the pump laser from the optical resonance frequency, the coefficient $G$ describes the shift of the optical resonance per mechanical displacement, and finally, $m, \Omega_{m}$ and $\Gamma_{m}$ respectively represent the effective mass, resonance frequency and the decay rate of the mechanical resonator. In addition, the output photon flux $\left|s_{\text {out }}\right|^{2}$ is related to the input via $s_{\text {out }}=s_{\text {in }}-\sqrt{\kappa_{e}} a$.
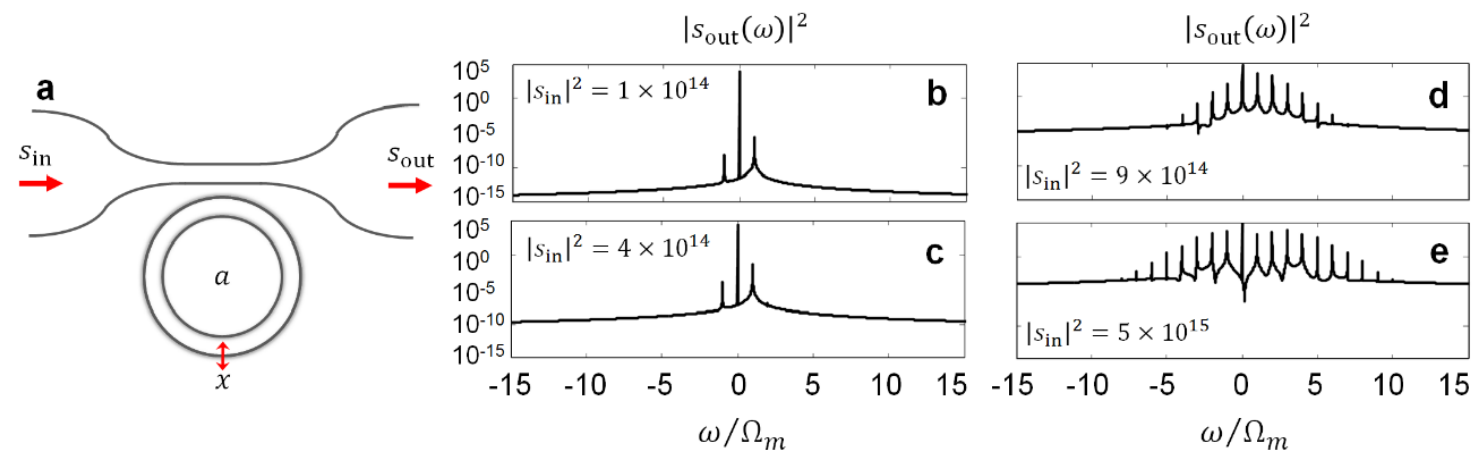

Fig. 1. (a) Schematic representation of a microring resonator cavity optomechanical system involving a whispering gallery traveling optical mode coupled to a radially beating mechanical mode, and (b-e) the optomechanically-induced frequency comb generated at different input powers. In these simulations, we consider a microtoroid cavity with $\kappa / 2 \pi=2 \kappa_{e} / 2 \pi=10 \mathrm{MHz}, \Omega_{m} / 2 \pi=50 \mathrm{MHz}, \Gamma_{m} / 2 \pi=50 \mathrm{KHz}$, $G / 2 \pi=6 \mathrm{GHz} / \mathrm{nm}$, and $m=6 \mathrm{ng}$. In addition, the system is driven in the extreme blue-detuned regime i.e., $\Delta=\Omega_{m}$. 
According to Eqs. (1), coupling to the mechanical degree of freedom induces a time-dependent cubic nonlinearity for the optical mode. Under steady-state conditions, these equations can be reduced to a single equation, namely: $\left(i\left(\Delta+\gamma\left|a_{0}\right|^{2}\right)-\kappa / 2\right) a_{0}+\sqrt{\kappa_{e}} s_{\text {in }}=0$, where $\gamma=\hbar G^{2} / m \Omega_{m}^{2}$ represents the effective steady-state cubic nonlinearity coefficient. At low input power levels, this equation describes the steady state behavior of the system, which results in the well-known optical bistability. However, by increasing the drive power two sidebands emerge at $\pm \Omega_{m}$ and, by further increasing the power, higher-order sidebands appear at $\pm 2 \Omega_{m}, \pm 3 \Omega_{m}$ and so on, until forming a frequency comb, as shown in Figs. 1(b-e). It is worth noting that, in addition to the drive power, the comb generation threshold also strongly depends on the frequency detuning parameter $\Delta$. Here, we consider the extreme blue-detuned regime, i.e., $\Delta=\Omega_{m}$, which drives the system towards parametric instabilities at much lower powers compared to the red-detuned regime.

Figure 2 depicts the power transmitted in different sidebands as a function of input power. The interplay of different sidebands can be further explored by decomposing the continuous-time field and position variables into different components as $a(t)=\sum_{n=-\infty}^{+\infty} a_{n} e^{i n \Omega_{m} t}$ and $x(t)=\sum_{n=-\infty}^{+\infty} x_{n} e^{i n \Omega_{m} t}$ where $a_{n}$ and $x_{n}$ represent slowly varying modal amplitudes of different sidebands while given that $x(t)$ is real, the positive and negative mechanical sidebands are related via $x_{-n}=x_{n}^{*}$. By using this relation in Eqs. (1), and after decomposing terms varying at similar rates, the dynamical equations (1) can be converted to a coupled set of nonlinear equations describing various sidebands.

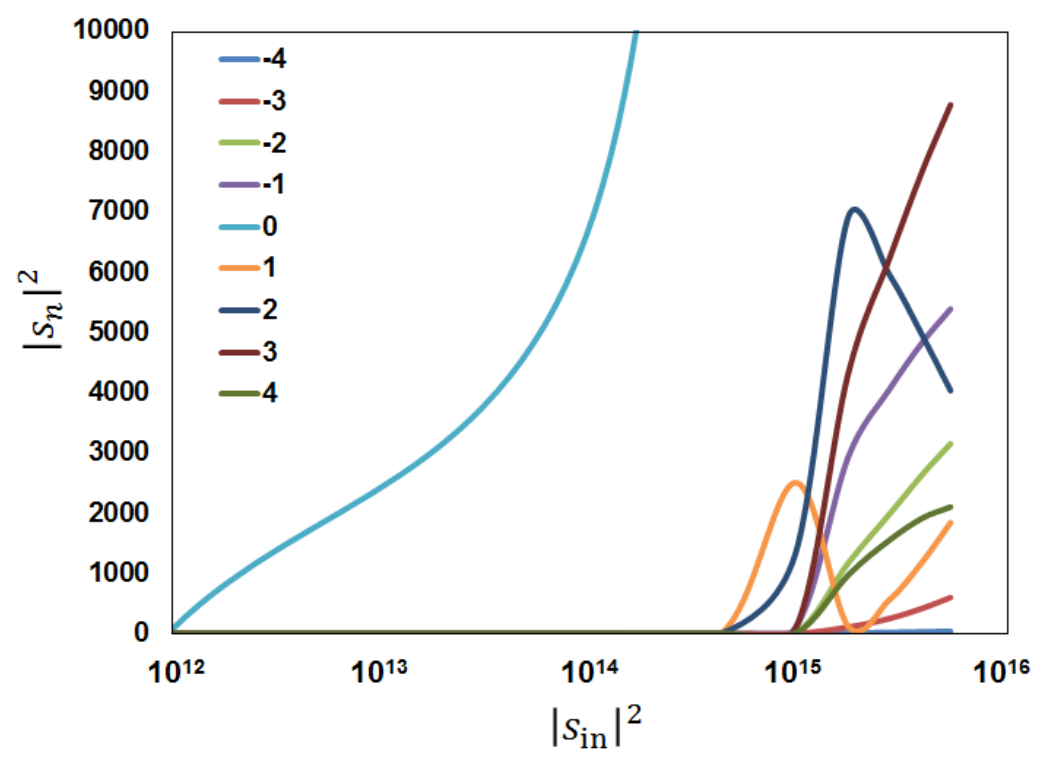

Fig. 2. Power transmitted in different sidebands $\left|s_{n}\right|^{2}$ versus the drive photon flux $\left|s_{\text {in }}\right|^{2}$. All parameters are the same as in Fig. 1.

In conclusion, we have studied the formation of optical frequency combs in a single-mode cavity optomechanical system. In our future efforts, we plan to explore the possibility of extending this analysis to multimode systems where multiple optical modes can be exploited to achieve broadband optomechanicallyinduced frequency combs.

\section{References}

[1] M. Aspelmeyer, T. J. Kippenberg, and F. Marquardt, Rev. Mod. Phys. 86, 1391 (2014).

[2] J. T. Hill, A. H. Safavi-Naeini, J. Chan, and O. Painter, Nat. Commun. 3, 1196 (2012).

[3] P. B. Deotare, I. Bulu, I. W. Frank, Q. Quan, Y. Zhang, R. Ilic, and M. Loncar, Nat. Commun. 3, 846 (2012).

[4] Z. Shen, Y. L. Zhang, Y. Chen, C. L. Zou, Y. F. Xiao, X. B. Zou, F.-W. Sun, G.-C. Guo, and C. H. Dong, Nat. Photon. 10, 657 (2016).

[5] F. Ruesink, M.-A. Miri, A. Alù, and E. Verhagen, Nat. Commun. 7, 13662 (2016).

[6] K. Fang, J. Luo, A. Metelmann, M. H. Matheny, F. Marquardt, A. A. Clerk, and O. Painter, arXiv:1608.03620 (2016). 